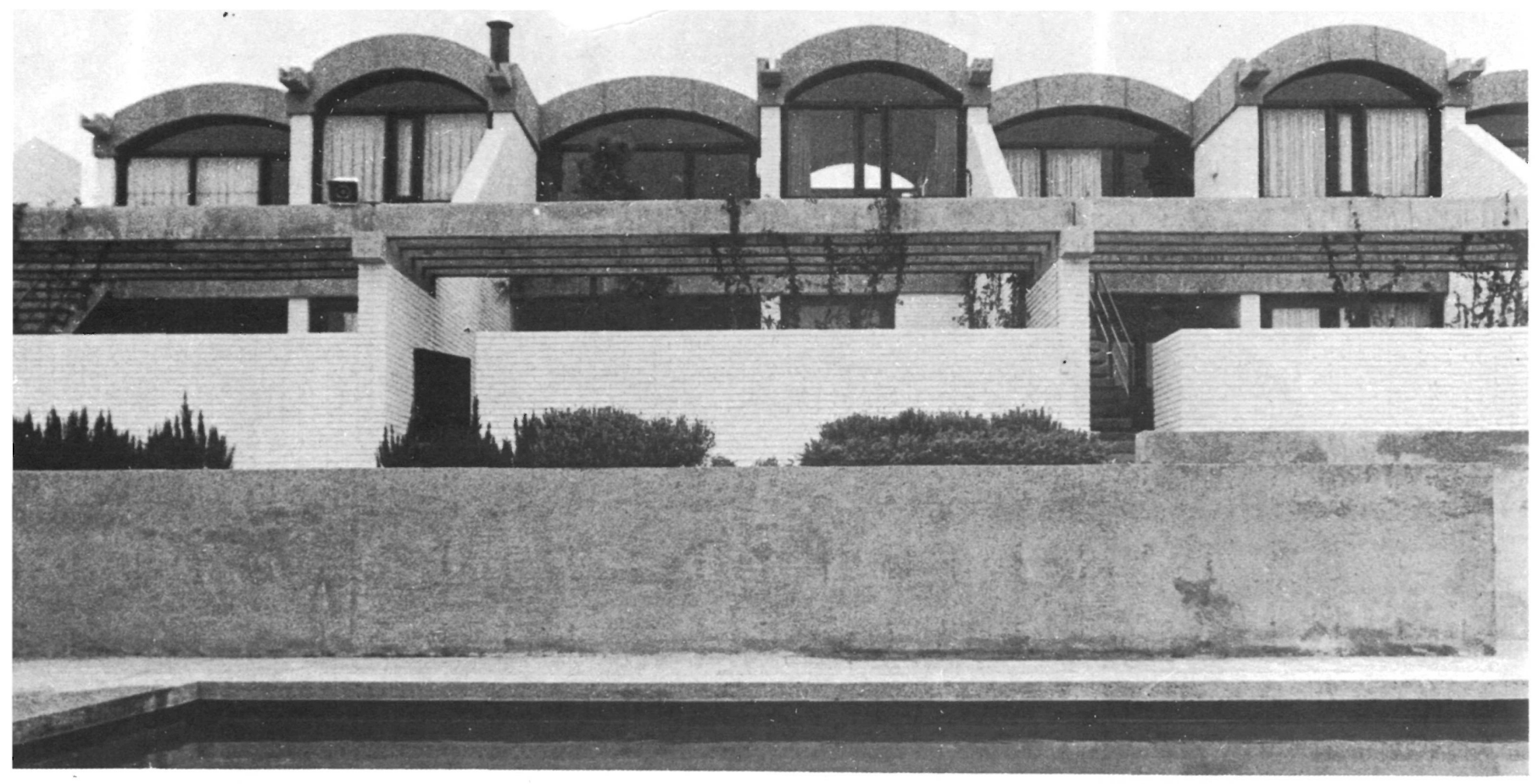

\title{
Grupo de viviendas unifamiliares adosadas, en Bellpuig \\ Lérida-España
}

Salvador Margarit Barcón, arquitecto

$161-207$

Antonio Consuegra Simón, aparejador

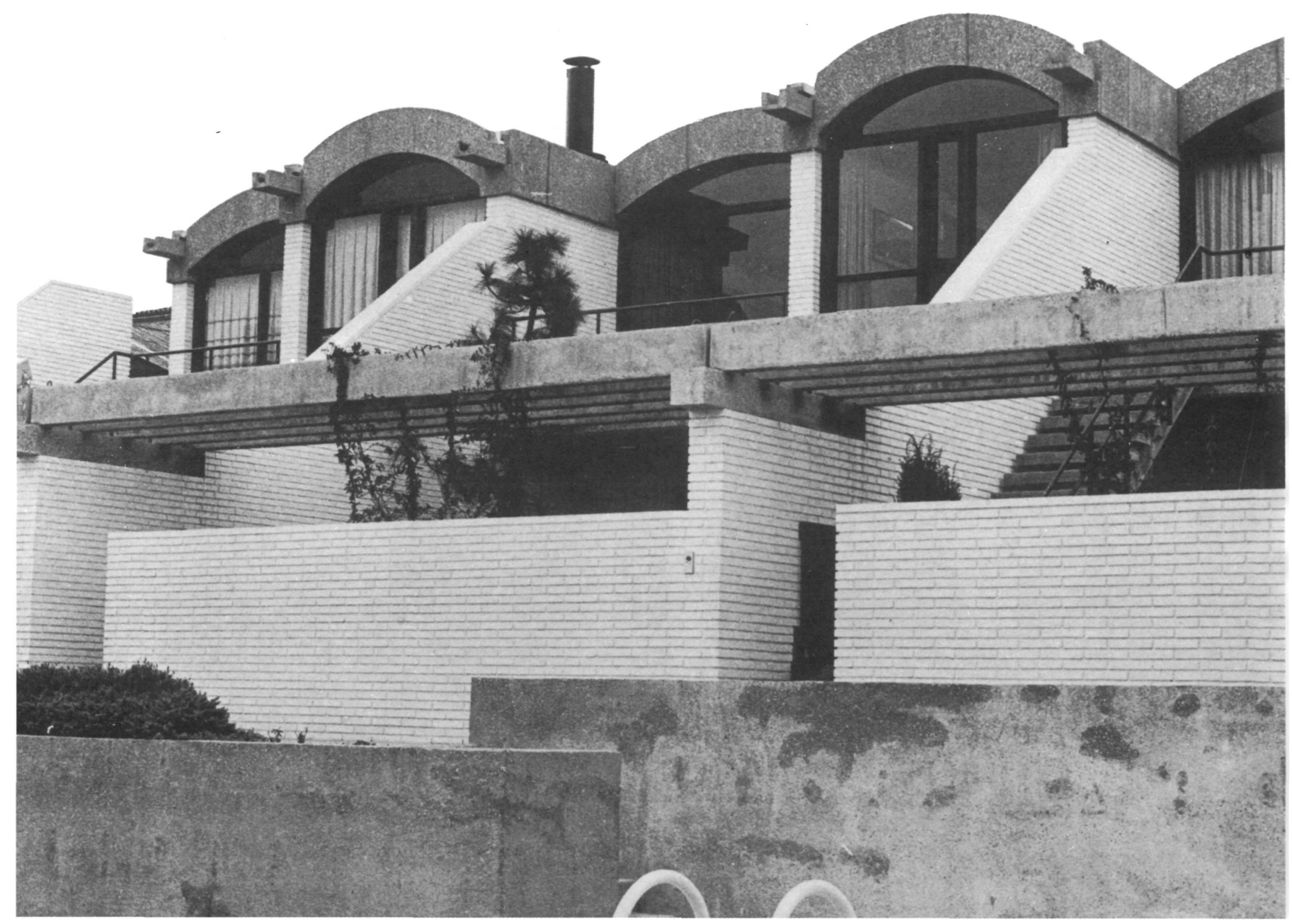



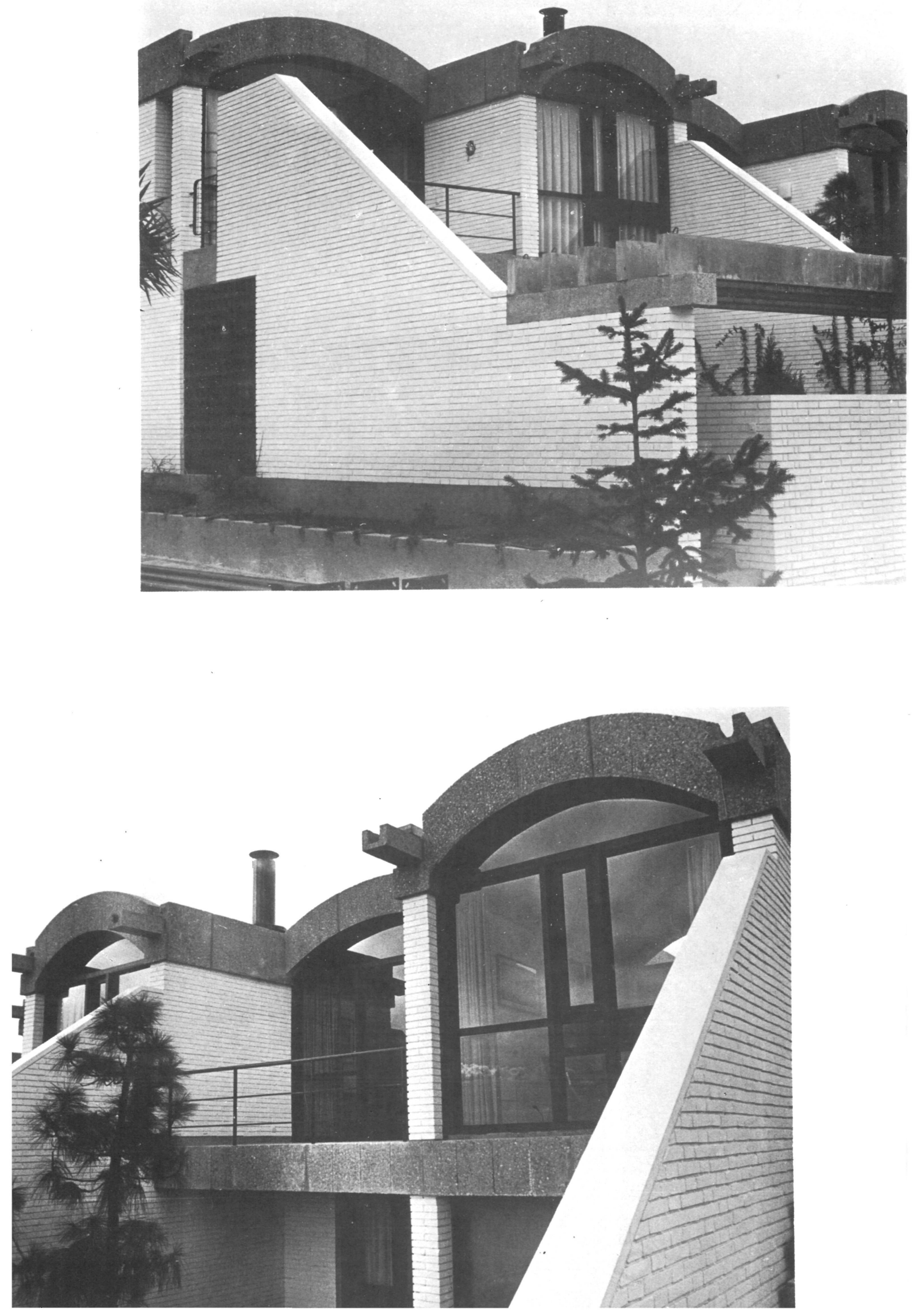

(c) Consejo Superior de Investigaciones Científicas Licencia Creative Commons 3.0 España (by-nc) 
Proyectado, hace algún tiempo, como residencias para el personal directivo de la empresa de prefabricados de hormigón G.M.P.

El proyecto original preveía la construcción de ocho viviendas adosadas y un edificio de apartamentos con 40 unidades. Unicamente las primeras han sido llevadas a término.

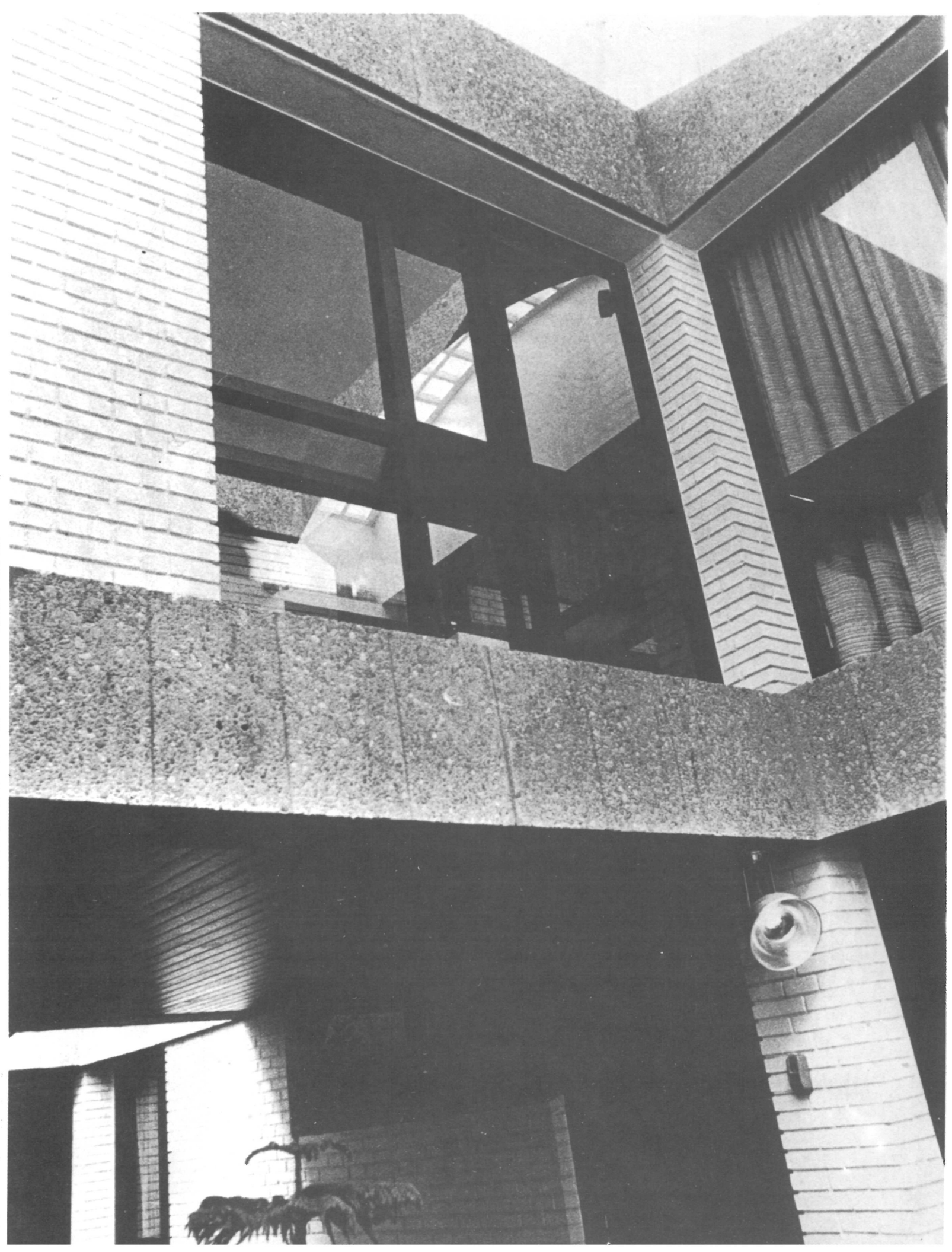




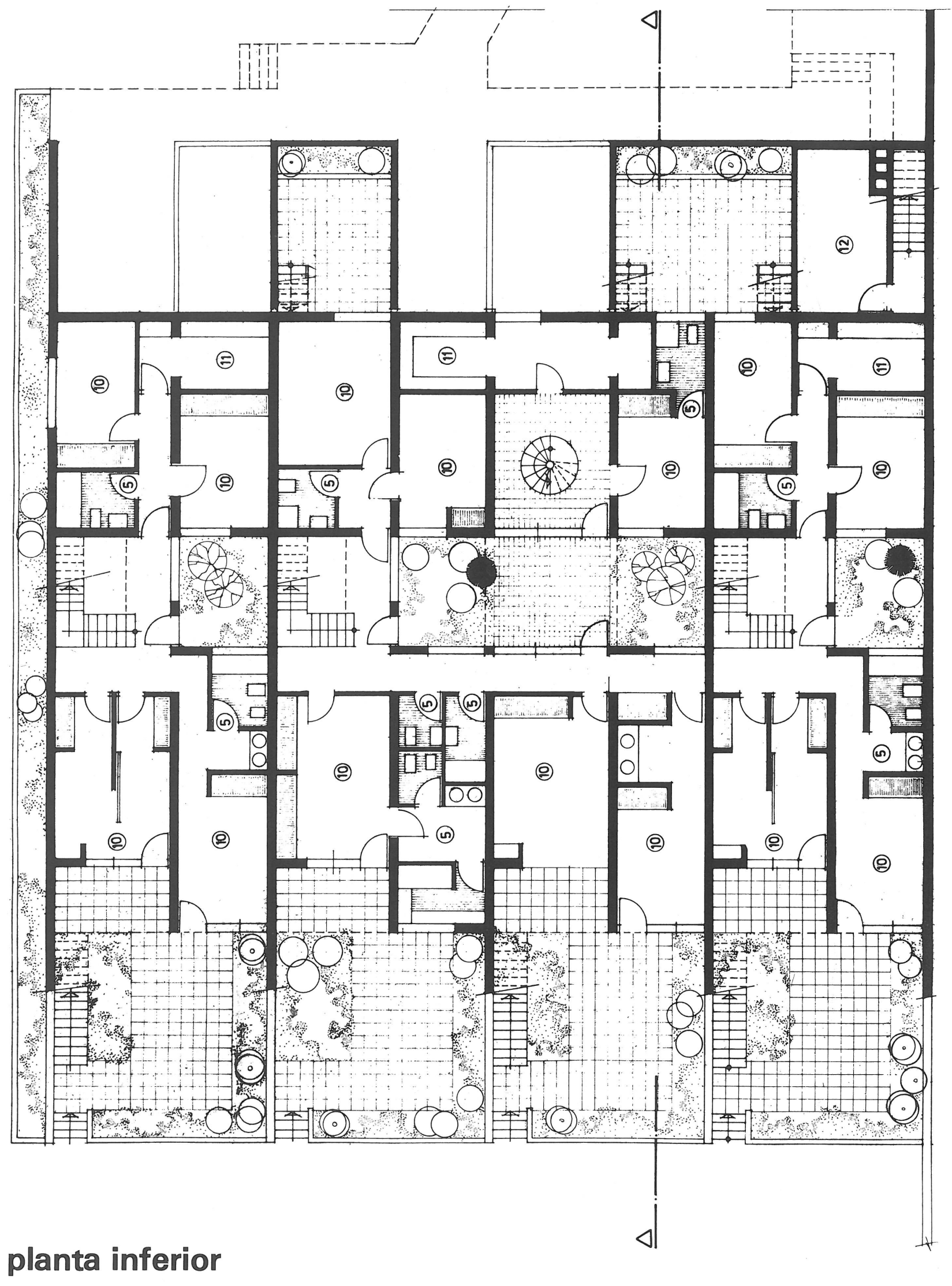




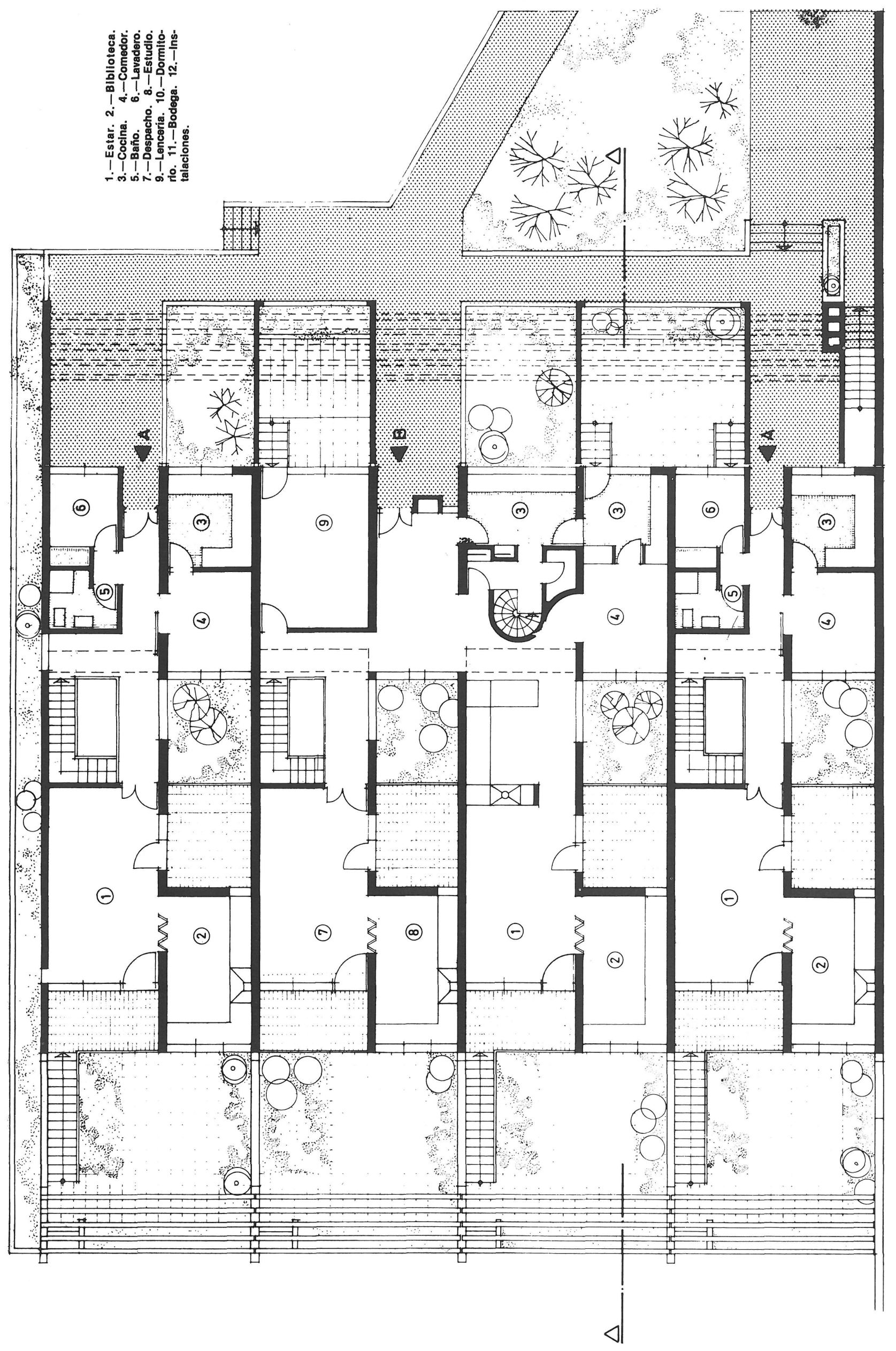

\begin{tabular}{l}
0 \\
0 \\
0 \\
0 \\
0 \\
0 \\
0 \\
0 \\
0 \\
0 \\
10 \\
4 \\
10 \\
0 \\
\hline 0
\end{tabular} 


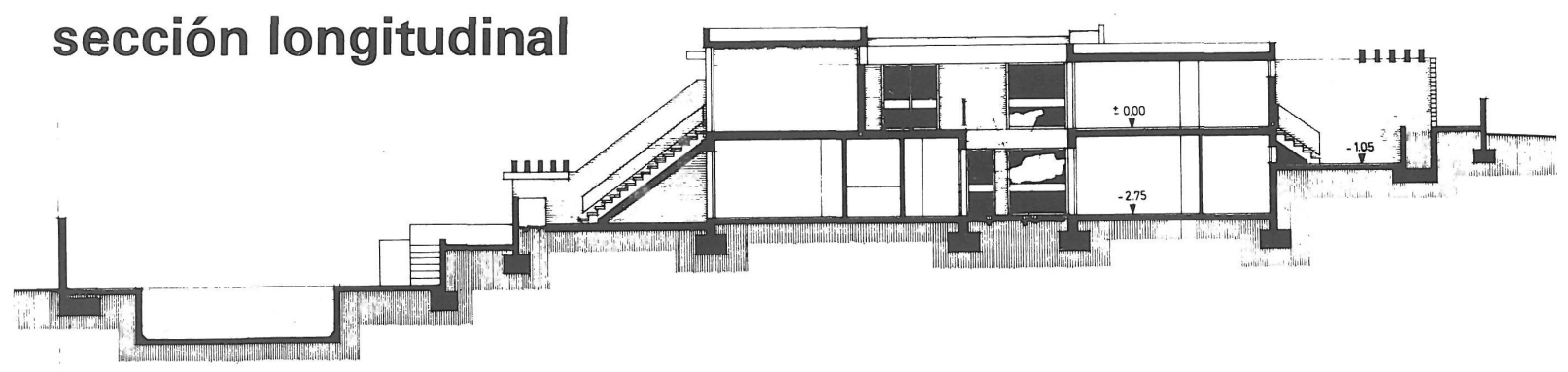

El terreno entre dos calles, ocupado antiguamente por una fábrica, presentaba un desnivel de casi una planta consolidado por un muro existente al que se adosaron las viviendas.

Los almacenes y edificaciones industriales antiguos de la población están, en gran parte, cubiertos con bóvedas catalanas. La posibilidad de contar con artesanos experimentados en este tipo de construcciones animó a los autores del proyecto a utilizar la bóveda catalana como tipo de cobertura y elemento de configuración de espacios y expresión formal.

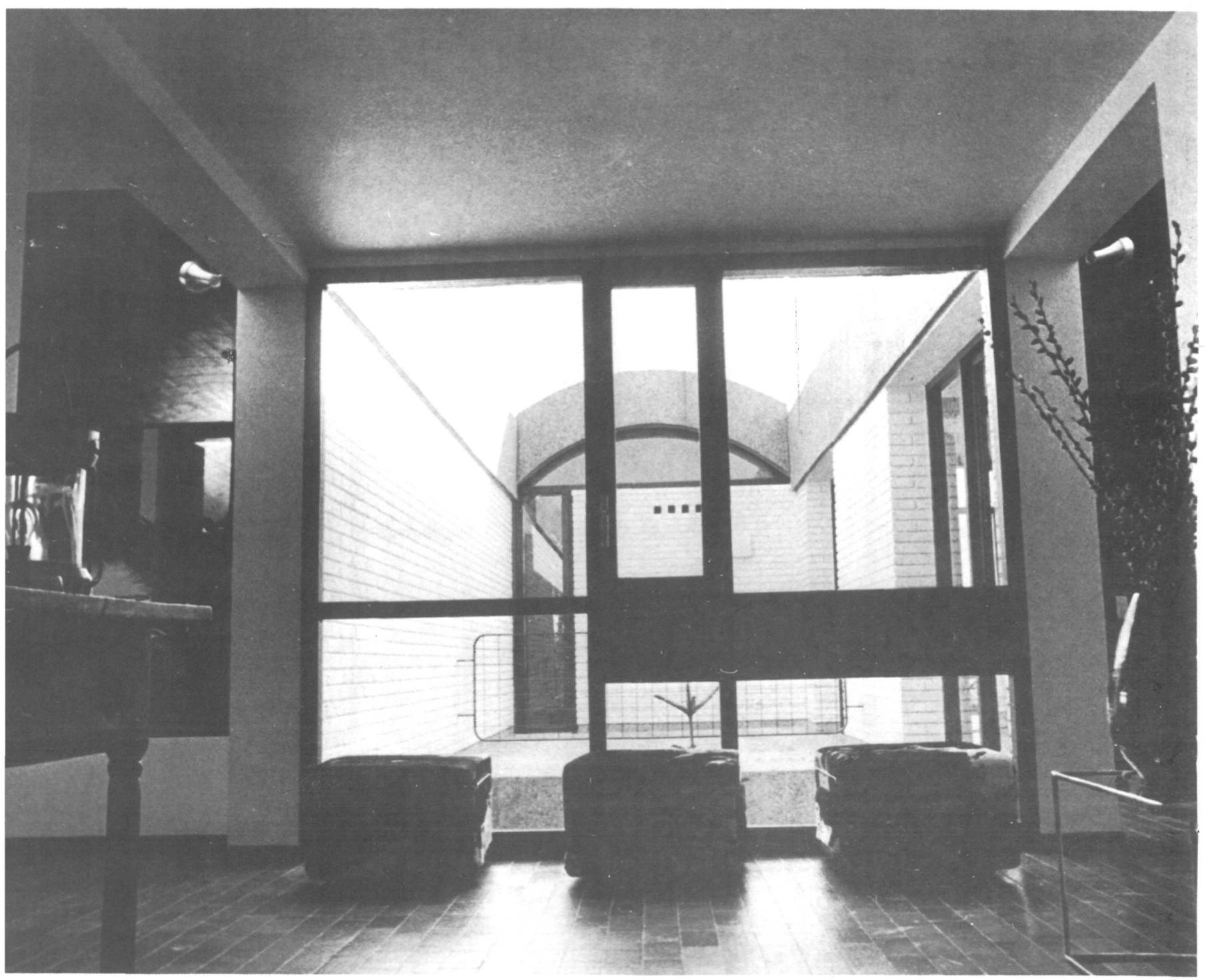



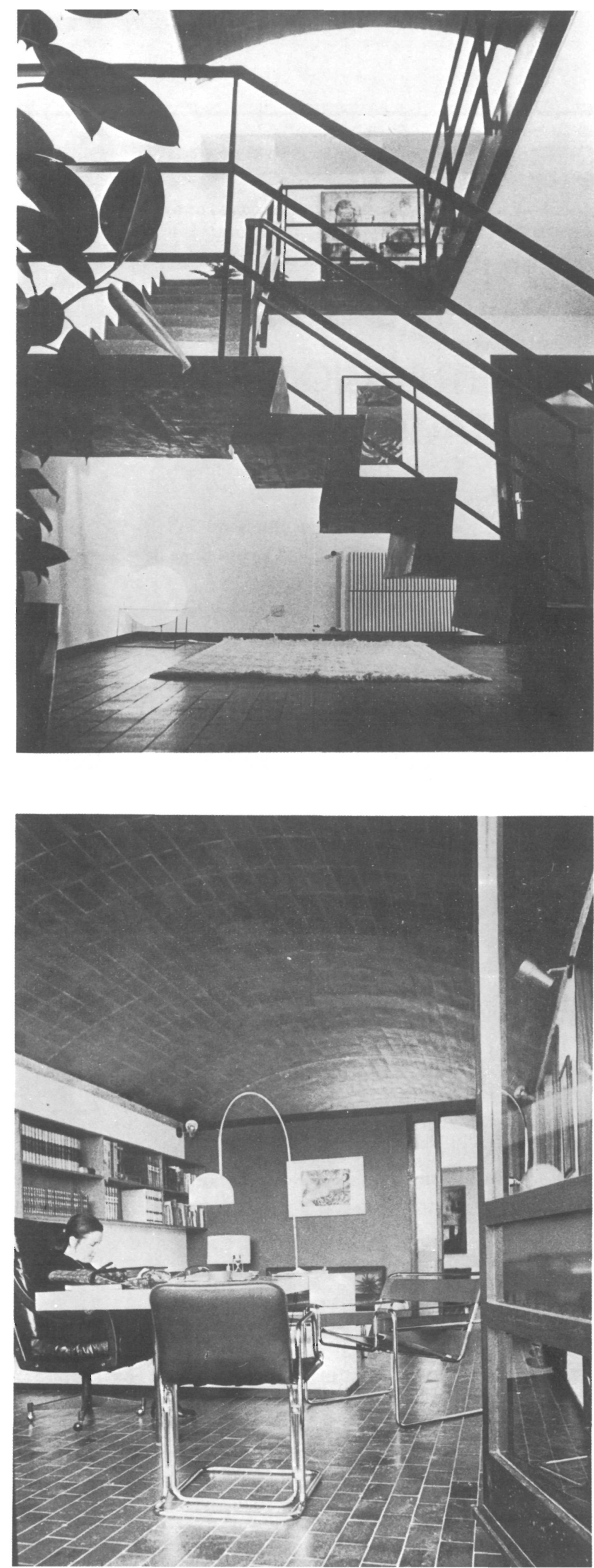

\section{résumé}

GROUPE DE LOGEMENTS INDIVIDUELS ADOSSES, A BELLPUIG LERIDA - ESPAGNE

Salvador Margarit Barcón, architecte Antonio Consuegra Simón,

aide-architecte

Le projet original, conçu il y a quelque temps comme des résidences pour les cadres de l'entreprise d'éléments préfabriqués en béton G.M.P., prévoyait la construction de huit logements adossés et d'un bâtiment pour 40 appartements. Seuls les logements adossés ont été construits.

Le terrain entre deux rues, occupé jadis par une usine, présentait un dénivellement de presque un niveau consolidé par un mur existant auquel les logements ont été adossés.

Les magasins et les bâtiments industriels anciens de la ville sont, en grande partie, couverts de voûtes à la catalane. La possibilité de compter sur des artisans expérimentes en ce type de construction a anim les auteurs du projet à utiliser la voûte à la catalane comme un type de couverture e comme un élément de configuration d'espaces et d'expression formelle.

\section{summary}

TOWNHOUSES - BELLPUIG, LERIDA

Salvador Margarit Barcón, Architect Antonio Consuegra Simón, Assistant Architect

This group was designed some time as a housing for the managerial staff of the G.M.P. concrete prefab manufacturing company.

The original design comprised eight townhouses and a tower with 40 flats, but only the townhouses have been built.

The property, a strip between two streets formerly occupied by a factory, presents a sharp drop, the higher ground supported by a retaining wall against which the back of the townhouses are abutted.

The old factories and warehouses in this industrial town are mostly built using the traditional "Catalonian vault" method. Tile availability of craftsmen experienced in this type of construction encouraged the designers to make use of this kind of vaulted roofing as a formal element to define the different areas.

\section{zusammenfassung}

GRUPPE VON EINFAMILIEN-REIHENHAUSERN IN BELLPUIG-LERIDA

Salvador Margarit Barcón, Architekt Antonio Consuegra Simón, Statiker

Diese Gruppe wurde vor einiger Zeit als Wohnmöglichkeit für das Personal der Direktion der Firma G.M.P. - Betonfertigteile - entworfen.

Das Originalprojekt sah die Errichtung von acht Einfamilien-Reihenhäusern und einem Gebäude mit 40 Appartments vor, von denen nur die zuerstgenannten fertiggestellt wurden.

Das zwischen zwei Strassen liegende Das zwischen zwei strüer eine Fabrik Gelande, auf demen Höhenunterschied vo befand, wies einen Hoh fast einem Stockwerk auf, der die sich die

Die alten Fabriklager und Gëbaude weisen zum grössten Teil katalonische Gewölbe auf Die Möglichkeit einer Zusammenarbeit mit Handwerkern, die in dieser Bauart vie Erfahrung hatten, verleitete die Architekten dazu, diese katalonischen Gewölbe für alle Ueberdachungen und raumbildenden mente zu wählen. 